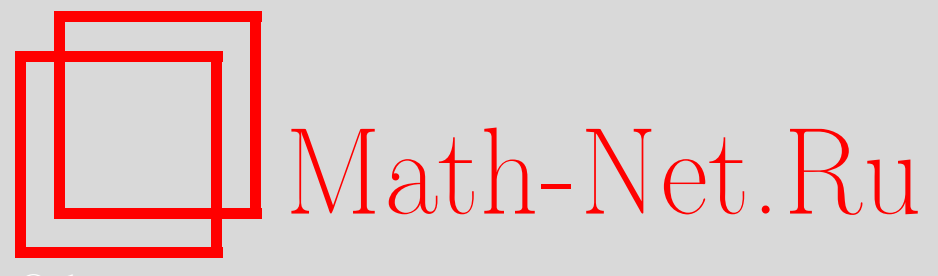

А. А. Черняк, Структурно-сложные системы с пороговой живучестью, Дискрет. матем., 1999, том 11, выпуск 4, 65-78

DOI: https://doi.org/10.4213/dm393

Использование Общероссийского математического портала Math-Net.Ru подразумевает, что вы прочитали и согласны с пользовательским соглашением http://www. mathnet.ru/rus/agreement

Параметры загрузки:

IP: 18.234 .156 .22

26 апреля 2023 г., 15:01:27

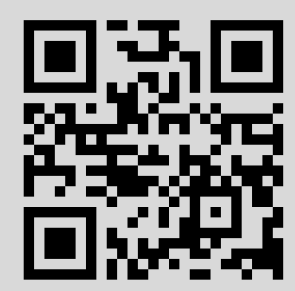




\title{
Структурно-сложные системы с пороговой живучестью
}

\author{
(C) 1999 г. А. А. Черняк
}

\begin{abstract}
Ранее была получена структурная характеризация сложных систем, моделируемых $K$-терминальными неориентированными сетями с пороговой живучестью. Проблема характеризации сложных систем, моделируемых $K$-терминальными ориентированными сетями с пороговой живучестью, оставалась открытой задачей. Решение этой задачи автоматически следует из полученной в данной статье характеризации $d c$-тривиальных графов (подкласса монотонных графов), имеющих пороговую живучесть, так как эти графы включают в себя в качестве специальных случаев все классические модели мультитерминальных сетей, применяемых для анализа надежности сложных систем. Доказано также, что в классе всех монотонных графов с пороговой живучестью задача распознавания разрешима за время, полиномиально зависящее от размерности графов и числа их минимальных путей.
\end{abstract}

\section{1. Введение}

Разнородность традиционных сетевых моделей надежности структурно-сложных систем послужила основным мотивом введения в [1] монотонных графов, допускающих в вершинах произвольную логику функционирования. В таких графах возможность прохождения сигнала через некоторую вершину определяется наборами сигналов, подающихся на входы этой вершины от остальных вершин, причем сами наборы соответствуют простым импликантам произвольной монотонной булевой функции. Распространение в [2, 3] теории доминирования на монотонные графы позволило выявить единую комбинаторную природу ранее известных результатов, касающихся надежности и доминирования ориентированных сетей.

Важным подклассом монотонных графов являются $d c$-тривиальные графы (см. раздел 2). Значение этих графов, в частности, в том, что они составляют граничный подкласс монотонных графов, включающий в себя в качестве специальных случаев все классические сетевые модели мультитерминальной надежности [4-6]. С другой стороны, как показано в [7], задача вычисления надежности является $N P$-трудной даже на множестве тех $d c$-тривиальных графов, в которых каждая вершина имеет не более двух входящих дуг, а число минимальных сечений пропорционально размерности графов. Это означает, что для таких графов задача̇ надежности не может быть решена алгоритмом, временная сложность которого ограничена полиномом от

3 Дискретная математика, т.11 №4 
числа их минимальных сечений, если класс $P$ полиномиальных и класс $N P$ недетерминированных полиномиальных алгоритмов не совпадают (даже в случае $P=N P$ существование такого алгоритма весьма проблематично [8]).

Наряду с надежностью существенное значение для структурно сложных систем, моделируемых сетями, имеют показатели живучести. Под живучестью сети подразумевается ее устойчивость к сохранению основных функций работоспособности при воздействии поражающих факторов внешней среды (см., например, [9]). В [10] была получена характеризация $K$-терминальных неориентированных сетей, живучесть которых обладает пороговым свойством. В таких сетях каждое ребро имеет определенную цену разрушения, а вся сеть теряет работоспособность (т.е. теряет связность между некоторыми вершинами из фиксированного множества $K$ ), если и только если сумма цены разрушения превосходит допустимый порог. Проблема характеризации $K$-терминальных ориентированных сетей, имеющих пороговую живучесть, оставалась открытой задачей.

Решение этой задачи автоматически следует из полученной в данной статье характеризации $d c$-тривиальных графов, имеющих пороговую живучесть. Приведенная в статье структурная характеризация позволила получить эффективный алгоритм распознавания таких графов. Доказано, что задача распознавания произвольных монотонных графов, имеющих пороговую живучесть, разрешима за время, полиноминально зависящее от размерности графов и числа их минимальных путей.

\section{2. Монотонные $(s, t)$-графы, предварительные сведения}

Все рассматриваемые ниже графы считаются ориентированными, $V G$ и $D G-$ множества вершин и дуг графа $G$ соответственно, $u w$ - дуга, направленная из $u$ в $w$, $u$ - начальная вершина дуги, $w$ - концевая вершина, $D^{+}(v, G)$ и $D^{-}(v, G)$ - множества дуг графа $G$, направленных в вершину $v$ и из вершины $v$ соответственно. Величина $\left|D^{+}(v, G)\right|$ называется степенью вершины $v$ в $G$. Последовательность дуг $e_{i}=v_{i} v_{i+1}, i=1, \ldots, n$, называется простой цепью или $\left(v_{1}, v_{n+1}\right)$-цепью, если вершины $v_{1}, \ldots, v_{n+1}$ различны. Если при этом допускается, что $v_{1}=v_{n+1}$, то такая последовательность дуг называется циклом. Скажем, что вершина $u$ достижима из вершины $v$ в $G$, если $v=u$ или в $G$ существует $(v, u)$-цепь. Если граф $G$ содержит хотя бы один цикл, то он называется циклическим, в противном случае $G-$ ациклический граф.

Инъективная функция $\varphi: V G \rightarrow Z^{+}$называется ранговой функцией графа $G$, если $\varphi(u)<\varphi(v)$ для любой дуги $u v \in D G$, при этом $\varphi(w)$ называется рангом вершины $w$. Граф $G$ имеет некоторую ранговую функцию, если и только если $G$ ациклический [11]. В дальнейшем будет считаться, что любой ациклический граф ранжирован, т.е. задан вместе со своей ранговой функцией.

Функция $w: D G \rightarrow R^{+}$называется весовой функцией графа $G$, а число $w(e)$ весом дуги $e$. Символ $\varphi \mid A$ обозначает ограничение функции $\varphi$ на множество $A$.

Граф $G$ называется $(s, t)$-графом, если

$$
s, t \in V G, \quad D^{-}(t, G)=D^{+}(s, G)=\varnothing, \quad D^{-}(v, G) \neq \varnothing, \quad D^{+}(v, G) \neq \varnothing
$$

для любой вершины $v \in V G \backslash\{s, t\}$. 
Дадим теперь определение монотонного $(s, t)$-графа. С каждой вершиной $v \neq s$ графа $G$ свяжем множество $\operatorname{th}(v, G)$ так называемых пороговых наборов вершины $v$ в $G$, подмножеств множества $D^{+}(v, G)$, обладающих следующими свойствами:

(a) ни один пороговый набор не содержится в другом;

(b) каждый элемент из $D^{+}(v, G)$ принадлежит пороговому набору из $\operatorname{th}(v, G)$.

Подграф $H$ графа $G$ называется монотонным подграфом, если $H-(s, t)$-граф и для каждой вершины $v \in V H \backslash\{s\}$ множество $\operatorname{th}(v, G)$ пороговых наборов вершины $v$ в $H$ индуцируется множеством $\operatorname{th}(v, G)$, т.е. $\operatorname{th}(v, H)$ состоит в точности из тех наборов в $\operatorname{th}(v, G)$, которые содержатся в $D^{+}(v, H)$ и $D^{+}(v, H)$ есть объединение этих наборов. Монотонный подграф $P$ графа $G$ называется минимальным путем в $G$, если $|\operatorname{th}(v, P)|=1$ для каждой вершины $v \in V P \backslash\{s\}$. Считается также, что все минимальные пути в $G$ ациклические (хотя сам граф $G$ может содержать циклы).

Определенный таким образом граф $G$ и его система минимальных путей называется монотонным $(s, t)$-графом. Сечение монотонного графа $G$ - это подмножество $\mathscr{E} \subseteq D G$, имеющее непустое пересечение с множеством дуг каждого минимального пути в $G$.

Замечание 1. Если в минимальных путях допустить циклы, то получим так называемые сильно-циклические монотонные графы.

Вершина $v$ называется $d$-вершиной в $G$, если $|\operatorname{th}(v, G)|>1$ и все пороговые наборы в $\operatorname{th}(v, G)$ одноэлементные. Вершина $v$ называется $c$-вершиной в $G$, если $v=s$ или $|\operatorname{th}(v, G)|=1$. Монотонный $(s, t)$-граф $G$ называется $d c$-тривиальным (монотонным) $(s, t)$-графом, если любая его вершина является $d$-вершиной или $c$-вершиной [7].

Рассмотрим теперь три классические графовые модели, используемые в задачах мультитерминальной надежности ориентированных сетей.

2.1. Напомним, что 2-терминальные графы [4] - это $(s, t)$-графы $G$, в которых минимальными путями являются $(s, t)$-цепи. Другими словами, $G$ - это $d c-$ тривиальный $(s, t)$-граф, в котором любая вершина, имеющая степень, большую 1 , является $d$-вершиной.

2.2. Исток- $K$-терминальные графы рассматривались в $[5,6]$. В таких графах $H$ задаются входная вершина $s$ (исток) и множество $K=\left\{v_{1}, \ldots, v_{r}\right\}$ выходных вершин, а минимальными путями являются $K$-деревья, т.е. минимальные ориентированные деревья с корнем $s$, содержащие $K$. Добавим к $H$ новую вершину $w$ и $r$ дуг $v_{i} w, i=1, \ldots, r$. Новый граф обозначим через $G$. Тогда $G$ - это $d c$-тривиальный $(s, t)$-граф, в котором любая вершина, отличная от $w$ и имеющая степень, большую 1 , является $d$-вершиной.

2.3. Оллтерминальный граф $H$ - это исток- $(V H \backslash s)$-терминальный граф $[5,6]$. Пусть $V H=\left\{s, v_{1}, \ldots, v_{n}\right\}$. Тогда минимальными путями в $H$ являются остовные деревья с корнем $s$. Добавим к $H$ новую вершину $w$ и $n$ дуг $v_{i} w, i=1, \ldots, n$. Полученный граф обозначим $G$ и назовем его оллтерминальным монотонным $(s, t)$ графом. Очевидно, $G$ - это $d c$-тривиальный $(s, t)$-граф, в котором $c$-вершина $w$ имеет степень $n$, а любая вершина, отличная от $w$ и имеющая степень, большую 1 , является $d$-вершиной.

Пусть $G$ - произвольный монотонный $(s, t)$-граф. Определим процедуру, применяемую к $G$ для построения усеченных минимальных путей графа $G$ в соответствии с некоторым правилом $\mathscr{L}$, и обозначаемую $\operatorname{Proc}(X, \mathscr{L})$. 
Процедура $\operatorname{Proc}(X, \mathscr{L}), X \subseteq V G$. Шаг 1. Обьявить вершины из $X$ помеченными. Положить

$$
V P:=\varnothing, \quad D P:=\varnothing, \quad u:=t
$$

и перейти к шагу 2.

Шаг 2. Объявить вершину $u$ помеченной. Выбрать в $\operatorname{th}(u, G)$ в соответствии с правилом выбора $\mathscr{L}$ пороговый набор $\mathrm{Ch}(u)$, множество начальных вершин дуг которого обозначить $B(u)$. Положить

$$
D P:=D P \cup \mathrm{Ch}(u), \quad V P:=V P \cup B(u) \cup\{u\}
$$

и перейти к шагу 3.

Шаг 3. Если все вершины в $V P$ помечены, то процедура завершена построением подграфа $P$ с множеством вершин $V P$ и дуг $D P$. Если не все вершины в $V P$ помечены, то выбрать среди них произвольную вершину $u$ (если $G$ ациклический, то выбрать вершину наибольшего ранга) и перейти к шагу 2.

Лемма 1 ([1]). Пусть $G$ - монотонный $(s, t)$-граф. Тогда любой его минималъный путь может быть построен некоторой прочедурой $\operatorname{Proc}(s, \mathscr{L})$. Eсли $x$ тому же $G$ ачиклический, то любая прочедура вида $\operatorname{Proc}(s, \mathscr{L})$ строчт минимальный путь графа $G$.

В дальнейшем неоднократно используется следующее правило выбора, обозначаемое через $\mathscr{L}(f, R)$ (здесь $R-$ минимальный путь в $G, f \notin D R, f=u w)$ :

- если $z \neq v$, то $C h(z):=D^{+}(z, R)$,

- если $z=v$, то $C h(z)$ должно содержать дугу $f$.

\section{3. Свойства $d c$-тривиальных графов с пороговой живучестью}

Пусть $G$ - произвольный монотонный $(s, t)$-граф. Скажем, что $G$ обладает пороговой живучестью, если существует весовая функция $w$ графа $G$ и число $b>0$ такие, что

$$
\sum_{f_{i} \in \mathscr{E}} w\left(f_{i}\right) \geqslant b,
$$

если и только если $\mathscr{E}$ - сечение в $G$. При этом пара $(w, b)$ называется пороговой для $G$. На рис. 1 приведен пример монотонного $(s, t)$ графа $G$ с пороговой живучестью, где $t, v, u-c$-вершины, $w-d$-вершина, веса дуг даны на рисунке, $b=2$. Если вершину $v$ объявить $d$-вершиной, то $G$ теряет свойство пороговой живучести.

Пусть $G$ - ациклический $d c$-тривиальный $(s, t)$-граф, $v_{1}, \ldots, v_{r}$ - множество его $d$-вершин, занумерованных в порядке возрастания их рангов. Граф $G$ назовем каркасным, если любая его $\left(v_{1}, t\right)$-цепь для каждого $i=2, \ldots, r$ содержит некоторую фиксированную дугу $g_{i}=u_{i} v_{i}$ из $D^{+}\left(v_{i}, G\right)$. Для каркасного графа $G$ определим процедуру $\operatorname{Red}(f, e, T, Q)$.

Пусть $Q$ - минимальный путь, содержащий все $d$-вершины каркасного графа $G$, $e \in D^{+}\left(v_{1}, Q\right), \quad f \in D^{+}\left(v_{1}, G\right) \backslash D^{+}\left(v_{1}, Q\right), \quad e=u v_{1}, \quad f=w v_{1}, \quad w \in V Q$, $T-$ минимальный путь, построенный процедурой $\operatorname{Proc}(s, \mathscr{L}(f, Q))$. 


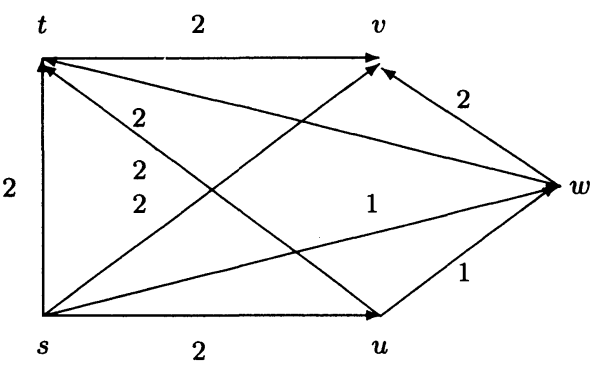

Pис. 1.

Процедура $\operatorname{Red}(f, e, T, Q)$. Шаг 1. Положить

$$
V F:=\left\{u, v_{1}\right\}, \quad D F:=\{e\}
$$

Назвать вершину $v_{1}$ опорной и перейти к шагу 2.

Шаг 2. Среди вершин $V F$, не являющихся внутренними и опорными, выбрать вершину $z$ наибольшего ранга и перейти к шагу 3. Если такой вершины нет, то перейти к шагу 5.

Шаг 3. Если $z \in V T$, то объявить вершину $z$ опорной и перейти к шагу 2. Если $z \notin V T$, то объявить $z$ внутренней вершиной и перейти к шагу 4 .

Шаг 4. Положить

$$
\begin{aligned}
& V F:=V F \cup\left\{\text { множество начальных вершин дуг из } D^{+}(z, Q)\right\} \\
& D F:=D F \cup D^{+}(z, D) .
\end{aligned}
$$

Перейти к шагу 2.

Шаг 5. Обозначить через $H$ подграф, полученный из $G$ удалением всех внутренних вершин из $V F$ и всех дуг множества $D F$ (называемого редуцируемой частью). Завершить процедуру.

Процедура $\operatorname{Red}(f, e, T, Q)$ называется редукцией, примененной к $G$, если для каждой внутренней вершины $z$ из $V F$

$$
D^{-}(z, G) \backslash D^{-}(z, Q)=\varnothing \text {. }
$$

При этом $H$ будет называться результатом редукции, $v_{1}$ опорной вершиной редукции. Очевидно,

$$
\{f\} \cup D Q=D T \cup D F
$$

Пусть

$$
\tau_{i}=\operatorname{Red}\left(f_{i}, e_{i}, T_{i}, Q_{i}\right), \quad i=1,2 .
$$

Редукцию $\tau_{2}$ назовем смежной с редукцией $\tau_{1}$, если $\tau_{2}$ применяется к результату редукции $\tau_{1}, Q_{2}=T_{1}$ и редуцируемая часть редукции $\tau_{2}$ содержит дугу $f_{1}$. Последовательность редукций $\Omega=\tau_{1}, \ldots, \tau_{r}$ назовем цепочкой редукций, если $\tau_{i}$ смежна с $\tau_{i-1}, i=2, \ldots, r$. Максимальную подпоследовательность редукций в цепочке, 
имеющих общую опорную вершину, назовем звеном цепочки $\Omega$. Скажем, что $\Omega$ применяется к $G$, если $\tau_{1}$ применяется к $G$. Результатом цепочки $\Omega$, примененной к $G$, считается результат редукции $\tau_{r}$. Если результатом некоторой цепочки редукций $\Omega$, примененной к $G$, является минимальный путь графа $G$, то цепочка $\Omega$ называется редуцирующей $G$, а $G$ называется тотально редуцируемым.

Теорема 1. Пустъ $G-d c-$ ривиальный $(s, t)$-граф. Тогда $G$ обладает пороговой живучестью, если и только если $G$ - каркасный тотально редуцируемый граф.

Доказательство. Докажем достаточность. Пусть $\Omega=\Omega_{1}, \ldots, \Omega_{s}$ - цепочка, редуцирующая граф $G$, где $\Omega_{k}=\tau_{1}^{k}, \ldots, \tau_{n_{k}}^{k}$ - звено цепочки $\Omega, k=1, \ldots, s$,

$$
\tau_{i}^{k}=\operatorname{Red}\left(e_{i+1}^{k}, e_{i}^{k}, T_{i+1}^{k}, T_{i}^{k}\right),
$$

$D F_{i}^{k}$ - редуцируемая часть редукции $\tau_{i}^{k}, r_{i}^{k}=\left|D F_{i}^{k}\right|$.

Рекуррентно зададим следующую систему целых чисел:

$$
\begin{aligned}
q_{1}^{1} & =1, \quad p_{1}^{k}=r_{1}^{k} q_{1}^{k}, \quad q_{i}^{k}=q_{i-1}^{k}+p_{i-1}^{k}, \quad i=2, \ldots, n_{k}+1, \\
q_{1}^{k+1} & =q_{n_{k}+1}^{k}, \quad b=q_{n_{s}+1}^{s}, \\
p_{i}^{k} & = \begin{cases}\left(r_{i}^{k}-1\right) q_{i}^{k}+2 p_{i-1}^{k}, & \text { если } r_{i}^{k}>1, \\
p_{i-1}^{k}, & \text { если } r_{i}^{k}=1, \quad i=2, \ldots, n_{k} .\end{cases}
\end{aligned}
$$

Определим весовую функцию $w$ графа $G$, полагая

$$
w\left(f_{j}\right)= \begin{cases}q_{1}^{1}, & \text { если } f_{j} \in D F_{1}^{1}, \\ q_{1}^{k}, & \text { если } f_{j} \in D F_{1}^{k} \backslash\left\{e_{n_{k-1}+1}^{k-1}\right\}, k=2, \ldots, s, \\ q_{i}^{k}, & \text { если } f_{j} \in D F_{i}^{k} \backslash\left\{e_{i}^{k}\right\}, i=2, \ldots, n_{k}, k=1, \ldots, s, \\ p_{i}^{k}, & \text { если } f_{j}=e_{i+1}^{k}, i=1, \ldots, n_{k}, k=1, \ldots, s, \\ b, & \text { если } f_{j} \in D T_{n_{s}+1}^{s} \backslash\left\{e_{n_{s}+1}^{s}\right\} .\end{cases}
$$

Докажем, что пара $(w, b)$ является пороговой для $G$.

Предположим, что $\mathscr{E} \subseteq D G$ - сечение в $G$. Пусть $k$ - наибольший индекс такой, что для некоторого $i, 2 \leqslant i \leqslant n_{k}+1, e_{i}^{k} \notin \mathscr{E}$. Среди таких дуг $e_{i}^{k}$ выберем дугу $e_{m}^{k}$ с максимально возможным индексом $m$. Так как минимальный путь $T_{m}^{k}$ не содержит дуг из множества

$$
Y=\left\{e_{m+1}^{k}, \ldots, e_{n_{k}+1}^{k}, e_{2}^{k+1}, \ldots, e_{n_{k+1}+1}^{k+1}, e_{2}^{k+2}, \ldots, e_{2}^{s}, \ldots, e_{n_{s}+1}^{s}\right\},
$$

$e_{m}^{k} \notin \mathscr{E}$ и $D T_{m}^{k} \cap \mathscr{E} \neq \varnothing$, множество $D T_{m}^{k} \cap \mathscr{E}$ содержит дугу, вес которой не менее $q_{m}^{k}$, и эта дуга не принадлежит множеству $Y$. Отсюда,

$$
\begin{aligned}
\sum_{f_{j} \in \mathscr{E}} w\left(f_{j}\right) & \geqslant q_{m}^{k}+p_{m}^{k}+\ldots+p_{n_{k}}^{k}+p_{1}^{k+1}+\ldots+p_{n_{k+1}}^{k+1}+\ldots+p_{1}^{s}+\ldots+p_{n_{s}}^{s} \\
& =q_{n_{k+1}}^{k}+p_{1}^{k+1}+\ldots+p_{n_{k+1}}^{k+1}+\ldots+p_{n_{s}}^{s} \\
& =q_{1}^{k+1}+p_{1}^{k+1}+\ldots+p_{n_{s}}^{s}=q_{1}^{s}+p_{1}^{s}+\ldots+p_{n_{s}}^{s}=q_{n_{s+1}}^{s}=b .
\end{aligned}
$$

Предположим теперь, что $\mathscr{E}$ не является сечением, т. е. $D G-\mathscr{E}$ содержит множество дуг некоторого минимального пути $R$. Согласно выбору $k$ и $m$, справедливо 
включение $Y \subseteq \mathscr{E}$. Поэтому, в силу леммы 1 и определения цепочки редукций, $R$ совпадает с некоторым $T_{j}^{i}$, в котором либо $i<k$, либо $i=k, j \leqslant m$. В любом случае, $D T_{m}^{k} \backslash\left\{e_{m}^{k}\right\} \subseteq D R$, откуда, с учетом того, что $e_{m}^{k} \notin \mathscr{E}$,

$$
\mathscr{E} \cap D T_{m}^{k}=\varnothing \text {. }
$$

Но $D G \backslash D T_{m}^{k}=Y \cup \mathscr{E}_{m-1}^{k}$, где

$$
\mathscr{E}_{m-1}^{k}=\bigcup_{i=1}^{m-1} D F_{i}^{k} \cup \bigcup_{1 \leqslant j \leqslant k-1,1 \leqslant i \leqslant n_{j}} D F_{i}^{j}
$$

Отсюда,

$$
\sum_{f_{j} \in \mathscr{E}} w\left(f_{j}\right) \leqslant p_{m}^{k}+\ldots+p_{n_{k}}^{k}+p_{1}^{k+1}+\ldots+p_{n_{k+1}}^{k+1}+\ldots+p_{n_{s}}^{s}+\sum_{f_{j} \in \mathscr{E}_{m-1}^{k}} w\left(f_{j}\right) .
$$

Докажем индукцией по $m$ и $k$, что

$$
\sum_{f_{j} \in \mathscr{E}_{m-1}^{k}} w\left(f_{j}\right)<q_{m}^{k}
$$

Тогда, по аналогии с (1), будет следовать, что правая часть в (2) не превосходит $b$, что и завершит доказательство достаточности.

Если $k=1, m=2$, то

$$
q_{2}^{1}=q_{1}^{1}+p_{1}^{1}=1+r_{1}^{k}=1+\sum_{f_{j} \in D F_{1}^{1}} w\left(f_{j}\right) .
$$

Рассмотрим теперь три случая.

Пусть $m=2$. В этом случае

$$
\begin{aligned}
q_{2}^{k+1} & =q_{1}^{k+1}+p_{1}^{k+1}=q_{n_{k}+1}^{k}+\left|D F_{1}^{k+1}\right| q_{1}^{k+1} \\
& =q_{n_{k}+1}^{k}+q_{1}^{k+1}+\left|D F_{1}^{k+1} \backslash\left\{e_{n_{k}+1}^{k}\right\}\right| q_{1}^{k+1} \\
& =q_{n_{k}+1}^{k}+q_{n_{k}}^{k}+\left(p_{n_{k}}^{k}+\left|D F_{1}^{k+1} \backslash\left\{e_{n_{k}+1}^{k}\right\}\right| q_{1}^{k+1}\right) \\
& >\sum_{f_{j} \in \mathscr{E}_{n_{k}}^{k}} w\left(f_{j}\right)+\sum_{f_{j} \in D F_{1}^{k+1}} w\left(f_{j}\right)=\sum_{f_{j} \in \mathscr{E}_{1}^{k+1}} w\left(f_{j}\right) .
\end{aligned}
$$

Пусть теперь $m>2$ и $D F_{m-1}^{k}=\left\{e_{m-1}^{k}\right\}$. Тогда

$$
\begin{aligned}
q_{m}^{k} & =q_{m-1}^{k}+p_{m-1}^{k}=q_{m-1}^{k}+p_{m-2}^{k}=q_{m-1}^{k}+w\left(e_{m-1}^{k}\right) \\
& >\sum_{f_{j} \in \mathscr{E}_{m-2}^{k}} w\left(f_{j}\right)+w\left(e_{m-1}^{k}\right)=\sum_{f_{j} \in \mathscr{E}_{m-1}^{k}} w\left(f_{j}\right) .
\end{aligned}
$$

Наконец, пусть $m>2$ и $\left|D F_{m-1}^{k}\right|>1$. Тогда

$$
\begin{aligned}
q_{m}^{k} & =q_{m-1}^{k}+p_{m-1}^{k} \\
& =q_{m-1}^{k}+\left(r_{m-1}^{k}-1\right) q_{m-1}^{k}+2 p_{m-2}^{k}=q_{m-1}^{k}+p_{m-2}^{k}+\sum_{f_{j} \in D F_{m-1}^{k}} w\left(f_{j}\right) \\
& >\sum_{f_{j} \in \mathscr{E}_{m-2}^{k}} w\left(f_{j}\right)+\sum_{f_{j} \in D F_{m-1}^{k}} w\left(f_{j}\right)=\sum_{f_{j} \in \mathscr{E}_{m-1}^{k}} w\left(f_{j}\right) .
\end{aligned}
$$


Докажем теперь необходимость. Пусть $(w, b)$ - пороговая пара для $G$. Занумеруем дуги в $D G$ в порядке невозрастания их весов. Отметим вначале, что любой монотонный $(s, t)$-подграф $H$ графа $G$ обладает пороговой живучестью. Действительно, любое подмножество $\mathscr{E} \subseteq D H$ является сечением в $H$, если и только если $\mathscr{E} \cup(D G \backslash D H)$ - сечение в $G$. Поэтому пара $\left(w \mid D H, b-\sum_{f_{j} \in D G \backslash D H} w\left(f_{j}\right)\right)$ является пороговой для $H$.

Дальше в доказательстве неоднократно используется следующее утверждение.

Предложение 1. Пусть $\left(f_{r}, f_{k}, Q\right)$ - произволъная тройка, в которой $Q$ - минимальный путь в $G$,

$$
f_{r} \notin D Q, \quad f_{k} \in D Q, \quad r<k, \quad f_{r}=z w, \quad f_{k}=u v .
$$

Тогда $z, w \in V Q$ и любая $(v, t)$-цепь в $Q$ содержит дугу из $D^{+}(w, Q)$, а вершина $z$ не достижима из $v$ в $Q$.

Доказателъство предлоэсения. Обозначим через $R$ подграф $Q-f_{k}+f_{r}$. Предположим, что $R$ не содержит в качестве подграфов минимальных путей графа $G$. Тогда $D G \backslash D R$ - сечение в $G$. Но $w\left(f_{r}\right) \geqslant w\left(f_{k}\right)$. Поэтому множество $\left((D G \backslash D R) \backslash f_{k}\right) \cup f_{r}$ - также сечение в $G$, т. е. дополнительное к нему множество, которым как раз и является $D Q$, не должно содержать множества дуг какого-либо минимального пути. Получаем противоречие. Итак, $R$ содержит в качестве подграфа некоторый минимальный путь $T$ графа $G$. Это означает, что $z, w \in V Q$ и, следовательно, $T$ должен быть построен процедурой $\operatorname{Proc}\left(s, \mathscr{L}\left(f_{r}, Q\right)\right)$, примененной к графу $Q+f_{r}=R+f_{k}$ (см. лемму 1). Обозначим через $\operatorname{Reach}(v)$ множество всех вершин, достижимых из $v$ в $Q$. Так как $t \in \operatorname{Reach}(v)$ и все вершины из $\operatorname{Reach}(v)$, исключая $v$, являются $c$ вершинами в $Q+f_{r}$, ввиду определения $\operatorname{Proc}\left(s, \mathscr{L}\left(f_{r}, Q\right)\right)$, вершина $f_{k} \in D T$, если найдется хотя бы одна $(v, t)$-цепь в $Q$, не содержащая $w$. Получаем противоречие. По той же причине $z \notin \operatorname{Reach}(v)$. Предложения доказано.

Вернемся к доказательству теоремы. Докажем, что граф $G$ ациклический. Так как $G$ является $(s, t)$-графом, существует минимальный путь $Q$, содержащий дугу $f_{n}=u v$ наименьшего веса. Так как любая $d$-вершіина в $G$ имеет входящую дугу, не принадлежащую $Q$, в силу предложения 1 , все вершины из $G$ принадлежат $Q$ и любая $d$-вершина достижима из $v$ в $Q$, т. е. принадлежит $\operatorname{Reach}(v)$. Обозначим через $\varphi_{1}$ и $\varphi_{2}$ ранговые функции подграфов графа $Q$, индуцированных множествами вершин $\operatorname{Reach}(v)$ и $V G \backslash \operatorname{Reach}(v)$ соответственно. Без ограничения общности можно считать, что $\varphi_{1}(x)>\varphi_{2}(y)$ для любых $x \in \operatorname{Reach}(v), y \in V G \backslash \operatorname{Reach}(v)$. Обозначим через $\varphi_{3}$ такую функцию, что

$$
\varphi_{3}\left|\operatorname{Reach}(v) \equiv \varphi_{1}, \quad \varphi_{3}\right|(V G \backslash \operatorname{Reach}(v)) \equiv \varphi_{2} .
$$

Очевидно, $\varphi_{3}-$ ранговая функция для $Q$. Кроме того, для любой дуги $e=z w$ из $D G \backslash D Q$, в силу предложения 1 ,

$$
w \in \operatorname{Reach}(v), \quad z \in V G \backslash \operatorname{Reach}(v),
$$

т.е. $\varphi_{3}(w)>\varphi_{3}(z)$. Таким образом, $\varphi_{3}-$ ранговая функция для $G$.

Так как любая $(v, t)$-цепь $C$ в $G$ состоит из вершин, ранги которых превосходят ранг вершины $v$, граф $C$ не может содержать дуг, не принадлежащих $D Q$ (см. доказанное выше). Другими словами, $C$ является $(v, t)$-цепью в $Q$. Из предложения 1 теперь следует, что $G$ - каркасный граф. 
Обозначим через $f_{k}=z w$ дугу; имеющую наименьший вес среди дуг в $D G \backslash D Q$, а через $T$ минимальный путь, построенный процедурой $\operatorname{Proc}\left(s, \mathscr{L}\left(f_{k}, Q\right)\right)$. Применяя предложение 1 для тройки $\left(f_{k}, f_{n}, Q\right)$, заключаем, что $D T \backslash D Q=\left\{f_{k}\right\}$. Очевидно, любая $d$-вершина $y$ в $G$, отличная от $w$, имеет в $D^{+}(y, G)$ дугу $f_{s}$, не принадлежащую $D T$. В силу предложения 1 для тройки $\left(f_{s}, f_{k}, T\right)$, вершина $y$ достижима из $w$ в $T$ и, следовательно, $y$ достижима из $v$ в $Q$. Поэтому вершина $w-$ ближайшая к $v$ в графе $Q d$-вершина графа $G$. Рассмотрим два случая.

В первом случае $v=w$. Пусть $D F-$ редуцируемая часть $\operatorname{Red}\left(f_{k}, f_{n}, T, Q\right)$. Если существует внутренняя вершина $x \in V F$ такая, что

$$
D^{-}(x, G) \backslash D^{-}(x, Q) \neq \varnothing,
$$

то имеется дуга $f_{s}=x y$ такая, что $f_{s} \notin D T$ и $y-d$-вершина в $G$. Но тогда тройка $\left(f_{s}, f_{k}, T\right)$ противоречит предложению 1, ибо $x \notin V T$. Итак, $\tau_{1}=\operatorname{Red}\left(f_{k}, f_{n}, T, Q\right)$ - редукция. Пусть $H$ - результат этой редукции, а дуга $f_{r}$ имеет наименьший вес среди дуг в $D G \backslash(D T \cup D F)$. Так как $r<k$, в случае $\left|D^{+}(v, H)\right|>1$ применимы предыдущие рассуждения, а в случае $\left|D^{+}(v, H)\right|=1$ мы находимся в рамках применимости рассуждений, используемых ниже во втором случае. В любом случае доказано, что к $H$ применима редукция $\operatorname{Red}\left(f_{r}, f_{k}, R, T\right)$, которая смежна с $\tau_{1}$.

Во втором случае $v \neq w$. Обозначим через $f_{t}$ дугу из $D^{+}(w, Q)$. Если $t<k$, то в силу предложения 1 для тройки $\left(f_{t}, f_{k}, T\right)$ начальная вершина дуги $f_{t}$ должна принадлежать $V T$. Но все вершины $(v, w)$-цепи, кроме $w$, являются $c$-вершинами в $G$. Поэтому и $f_{n} \in D T$, что невозможно (см. доказательство предложения 1 , где в роли $f_{n}$ берется дуга $f_{k}$ и где показано, что $\left.f_{k} \notin D T\right)$.

Итак, $t>k$ и применимы рассуждения, использованные в первом случае, в силу которых к $G$ применима редукция $\tau_{1}=\operatorname{Red}\left(f_{k}, f_{t}, T, Q\right)$. Дальунейшие рассуждения аналогичны соответствующим рассуждениям первого случая.

Продолжая этот процесс, через конечное число шагов построим искомую редуцирующую цепочку. Теорема доказана.

Замечание 2. В общем случае возможно экспоненциально большое число цепочек, редуцирующих $d c$-тривиальный граф с пороговой живучестью. Однако приводимая ниже теорема 2 гарантирует эффективное построение некоторой канонической цепочки редукций такого графа, что автоматически означает эффективную разрешимость задачи распознавания $d c$-тривиальных графов с пороговой живучестью.

Приведем обозначения, используемые ниже в леммах 2 и 3 . Граф $G$ - каркасный $d c$-тривиальный $(s, t)$-граф; $v_{1}, \ldots, v_{r}-d$-вершины графа $G$, занумерованные в порядке возрастания их рангов; $C-\left(v_{1}, t\right)$-цепь, содержащая дуги $g_{i}=u_{i} v_{i}$ графа $G, i=2, \ldots, r ; \tau_{i}=\operatorname{Red}\left(e_{i+1}, e_{i}, Q_{i+1}, Q_{i}\right) ; P$ - подграф графа $G$, построенный процедурой $\operatorname{Proc}\left(\left\{s, v_{1}\right\}, \mathscr{L}(C)\right)$ в соответствии со следующим правилом выбора $\mathscr{L}(C)$ :

- если $z=v_{i}, i>1$, то $C h(z)=g_{i}$.

Так как $G$ - каркасный граф, подграф $P$ определяется однозначно.

Дугу $u v_{1}$ назовем вырожденной (невырожденной) относительно $P$, если $u \in V P$ $(u \notin V P)$.

Лемма 2. Пусть $G$ обладает пороговой живучестью $u \Omega=\tau_{1}, \ldots, \tau_{s}-$ редуцирующая его цепочка, в которой $D P \subseteq D Q_{1}$. Предположим, что $\tau_{i}$ - некоторая редукция первого звена в $\Omega$. Тогда вырожденность $e_{i}$ влечет вырожденность $e_{i+1}$, 
и если $e_{i+1}$ невырождена, то начальная вершина дуги $e_{i}$ достижима в $G$ из началвной дуги $e_{i+1}$.

Доказателъство. Если $D F_{i}$ - редуцируемая часть редукции $\tau_{i}$, то, ввиду вырожденности $e_{i}, D F_{i}=\left\{e_{i}\right\}$. Так как $D P \subseteq D Q_{1}$, справедливо включение $D P \subseteq D Q_{i}$, откуда следует, что $D Q_{i}=D P \cup\left\{e_{i}\right\}$. Но, по определению редукции, начальная вершина $w$ дуги $e_{i+1}$ принадлежит $V Q_{i}$. Отсюда, $w \in V P$, что означает вырожденность дуги $e_{i+1}$.

Докажем второе утверждение. Пусть $w$ - начальная вершина дуги $e_{i+1}$. По предположению, $w \notin V P$. Но по определению редукции $w \in V Q_{i}$ и $D Q_{i} \backslash D Q_{i+1}=D F_{i}$. Поэтому $w$ инцидентна некоторой дуге из $D F_{i}$, что и означает достижимость начальной вершины дуги $e_{i}$ из $w$. Лемма доказана.

Порогом вырожденности назовем число $t$ такое, что $t-1-$ наибольший индекс, при котором $e_{t-1}$ невырождена ( $e_{0}$ считаем невырожденной фиктивной дугой).

Из определения звена цепочки редукций непосредственно вытекает следующее утверждение.

Лемма 3. Пустъ $G$ обладает пороговой живучестъю и $\tau_{1}, \ldots, \tau_{r}$ - первое звено некоторой цепочки $\tau_{1}, \ldots, \tau_{s}$, редуцирующей $G$, причем $D P \subseteq D Q_{1}$. Пустъ $\theta-$ произволъная перестановка чисел $t, t+1, \ldots, r+1$. Тогда $\tau_{1}, \ldots, \tau_{t-2}, \tau_{t-1}^{\prime}, \tau_{\theta(t)}, \ldots, \tau_{\theta(r)}$, $\tau_{r+1}^{\prime}, \ldots, \tau_{s}-$ чепочка, редуцирующая $G$, где

$$
\begin{aligned}
\tau_{t-1}^{\prime} & =\operatorname{Red}\left(e_{\theta(t)}, e_{t-1}, Q_{\theta(t)}, Q_{t-1}\right), \quad t>1, \\
\tau_{\theta(i)} & =\operatorname{Red}\left(e_{\theta(i+1)}, e_{\theta(i)}, Q_{\theta(i+1)}, Q_{\theta(i)}\right), \\
\tau_{r+1}^{\prime} & =\operatorname{Red}\left(e_{r+2}, e_{\theta(r+1)}, Q_{r+2}, Q_{\theta(r+1)}\right) .
\end{aligned}
$$

В теореме 2 сохраняем смысл обозначений $C, P, v_{1}, \ldots, v_{r}, g_{2}, \ldots, g_{r}$. Кроме того, полагаем, что $D^{+}\left(v_{1}, G\right)=\left\{c_{1}, \ldots, c_{j+1}\right\}$, и дуги $c_{1}, \ldots, c_{j+1}$ занумерованы в порядке убывания рангов их начальных вершин.

Теорема 2. Пусть $G$ является dс-тривиальным $(s, t)$-графом. Тогда $G$ обладает пороговой живучестъю, если и толъко если $G$ - каркасный граф и $\propto$ применима редукция вида $\tau=\operatorname{Red}\left(c_{2}, c_{1}, R_{2}, R_{1}\right)$, где $D P \subseteq D R_{1}$, и результат редукции $\tau$ также обладает пороговой живучестъю.

Доказательство. Используем индукцию по числу дуг.

Докажем необходимость. В силу теоремы 1 , граф $G$ каркасный и к нему применима редуцирующая цепочка, начинающаяся с редукции $\tau_{1}=\operatorname{Red}\left(f_{k}, f_{m}, T, Q\right)$; здесь $T, Q, f_{k}, f_{n}, f_{t}$ имеют тот же смысл, что и в доказательстве теоремы 1 (при этом $m=n$ или $m=t$ в зависимости от ситуации, рассматриваемой в доказательстве теоремы 1). Очевидно, $D P \subseteq D Q$.

Предположим вначале, что $f_{m}$ вырождена. Тогда, в силу леммы 2 , все дуги из $D^{+}\left(v_{1}, G\right)$ вырождены. Из леммы 3 теперь следует, что существует цепочка, редуцирующая $G$, которая начинается с $\tau$.

Пусть теперь $f_{m}$ невырождена. Тогда, в силу леммы 2 , начальная вершина дуги $f_{m}$ имеет наибольший ранг среди всех начальных вершин невырожденных дуг, откуда, согласно выбору $c_{1}, f_{m}=c_{1}$. Аналогично, в случае невырожденности $f_{k}$, $f_{k}=c_{2}$, т. е. $\tau_{1}=\tau$. Если же $f_{k}$ вырождена, то из леммы 3 следует существование редуцирующей цепочки, начинающейся $\mathrm{c} \tau$. 
Докажем достаточность. Пусть $H$ - результат редукции $\tau$. Если $\left|D^{+}\left(v_{1}, G\right)\right| \geqslant 3$, то по индуктивному предположению (ибо $H$ обладает пороговой живучестью как монотонный $(s, t)$-подграф графа $G)$ к $H$ применима редукция $\operatorname{Red}\left(c_{3}, c_{2}, R_{3}, R_{2}\right)$, которая смежна с $\tau$, причем $D P \subseteq D R_{2}$.

Пусть $\left|D^{+}\left(v_{1}, G\right)\right|=2$. Обозначим через $P^{\prime}$ подграф графа $H$, построенный процедурой $\operatorname{Proc}\left(\left\{s, v_{2}\right\}, \mathscr{L}(C)\right)$. Если дуга $g_{2}$ невырождена относительно $P^{\prime}$, то ее начальная вершина $u_{2}$ имеет максимальный ранг среди начальных вершин других дуг из $D^{+}\left(v_{2}, G\right)$, невырожденных относительно $P^{\prime}$ (в доказательстве теоремы 1 показано, что $u_{i}$ имеет наибольший ранг среди всех начальных вершин дуг из $\left.D^{+}\left(v_{i}, G\right)\right)$. Поэтому, в силу индуктивного предположения, к $H$ применима редукция $\operatorname{Red}\left(x, g_{2}, R_{3}, R_{2}\right)$, которая смежна с $\tau$, причем $D P^{\prime} \subseteq D R_{2}$.

Итак, приведенные выше рассуждения применимы и к графу $H$. Поэтому, продолжая этот процесс, через конечное число шагов построим цепочку, редуцирующую граф $G$. Это и означает, в силу теоремы 1 , что $G$ обладает пороговой живучестью. Теорема доказана.

\section{4. Следствия}

Следствие 1. Задача распознавания $d c-$ тривиалъных графов $G$, обладающих пороговой живучестью, разрешима за время $O(|D G| \cdot|V G|)$.

Доказательство. Проверка ацикличности графа $G$, а также его ранжирование осуществимы за время $O(|D G|)[11]$. За это же время определяется $\left(v_{1}, v_{r}\right)$-цепь $C$ (если она существует), содержащая $d$-вершины графа $G$, а также подграф $P$ и порог вырожденности $t$. Таким образом, результат редукции $\tau$ (см. формулировку теоремы 2 ) можно определить за время $O(|D G|)$. Остальное следует из теоремы 2.

Следствие 2. Оллтерминалъный $(s, t)$-граф $G$ обладает пороговой живучестъю, если и только если он ациклический и имеет не более одной $d$-вершины.

Доказательство. Докажем необходимость. В силу теоремы 1 , граф $G$ каркасный. Наличие двух $d$-вершин $u$ и $v$ означало бы существование $(v, t)$-цепи, состоящей из единственной дуги $v t$ и не проходящей через вершину $u$ (считаем, что ранг $u$ больше ранга вершины $v$ ). Получаем противоречие.

Докажем достаточность. Граф $G$ является каркасным, так как имеет не более одной $d$-вершины. Если $d$-вершин нет, то все доказано. Пусть $v-d$-вершина в $G$, $D^{+}(v, G)=\left\{e_{1}, \ldots, e_{r}\right\}$. Обозначим через $Q$ произвольный минимальный путь в $G$, содержащий дугу $e_{1}$. По определению, $Q-$ остовный подграф. Положим

$$
\tau_{i}=\operatorname{Red}\left(e_{i+1}, e_{i}, Q-e_{1}+e_{i+1}, Q-e_{1}+e_{i}\right) \text {. }
$$

Непосредственно проверяется, что $\tau_{1}, \ldots, \tau_{r-1}$ - цепочка, редуцирующая $G$. Достаточность теперь следует из теоремы 1.

Пусть $H-(s, t)$-граф с ранговой функцией $\varphi$. Граф $H$ назовем арфа-графом, если $H$ содержит остовную $(s, t)$-цепь и для любых дуг $u v$ и $z w$, не принадлежащих этой цепи (такие дуги назовем хордами арфа-графа) либо

$$
\varphi(u) \leqslant \varphi(z), \quad \varphi(v) \geqslant \varphi(w)
$$


либо

$$
\varphi(u) \geqslant \varphi(z), \quad \varphi(v) \leqslant \varphi(w) .
$$

Следствие 3. Пороговой живучестью 2-терминалъный $(s, t)$-граф $G$ обладает, если и только если он является арфа-графом.

Доказательство. Проведем доказательство индукцией по числу дуг. Докажем необходимость. Так как минимальными путями графа $G$ являются $(s, t)$-цепи, утверждение очевидно, если $G$ - минимальный путь. Предположим теперь, что $G$ содержит $d$-вершины. Тогда, в силу теоремы 1 , существует цепочка, редуцирующая $G$. Пусть $\tau=\operatorname{Red}(f, e, T, Q)$ - первая редукция в этой цепочке. Тогда $Q$ - остовный минимальный путь, т.е. $Q$ - остовная $(s, t)$-цепь. Пусть $H$ - результат редукции $\tau$, $f=w v, D F$ - редуцируемая часть. Из определения редукции следует теперь, что $D F$ является $(w, v)$-цепью в $G$, ибо $D Q=(D T \backslash\{f\}) \cup D F$. Кроме того, из доказательства теоремы 1 следует, что для любой дуги $g=z x$ из $D G \backslash(D Q \cup f)$ ранг вершины $z$ меньше ранга вершины $v$. Но по предположению индукции, $H$ является арфа-графом с остовной цепью $D T$ (в арфа-графах остовная цепь определяется однозначно), в которой начальные вершины всех хорд предшествуют вершине $v$. Это означает, что $G$ - также арфа-граф с остовной цепью $D Q$, так как множество хорд в $G$ в точности состоит из хорд графа $H$ и дуги $f$.

Докажем теперь достаточность. Пусть $Q$ - остовная $(s, t)$-цепь арфа-графа $G$, $g=w v-$ хорда с минимальным расстоянием между вершинами $w$ и $v$ в цепи $Q, D^{+}(v, Q)=\{e\}$. Очевидно, к $G$ применима редукция $\operatorname{Red}(g, e, T, Q)$. При этом результат редукции $H$ также является арфа-графом с остовной $(s, t)$-цепью $D T$. Остальное следует из индуктивного предположения и теоремы 1.

Теорема 3. Задача распознавания монотонных $(s, t)$-графов, обладающих пороговой живучестъю, разрешима за время, полиномиалъно зависящее от их размерности и числа минимальных путей.

Доказательство. Отметим, что доказательство ацикличности графа $G$ в теореме 1 применимо к произвольному монотонному графу, обладающему пороговой живучестью: для этого в доказательстве достаточно только $d$-вершины заменить вершинами, отличными от $c$-вершин. Поэтому можно считать $G$ ранжированным монотонным $(s, t)$-графом. Следующий алгоритм порождает множество $\mathscr{P}=\left\{P_{1}, \ldots, P_{m}\right\}$ всех его минимальных путей.

Шаг 1. Объявить вершину $s$ помеченной. Положить $V P:=\varnothing, D P:=\varnothing, \operatorname{back}(t):=$ $\varnothing, u:=t$. Для каждой вершины $v \in V G \backslash\{s\}$ занумеровать все пороговые наборы в $\operatorname{th}(v, G)$ целыми числами от 1 до $|\operatorname{th}(v, G)|$. Положить $\operatorname{count}(v):=1$ для каждой вершины $v \in V G \backslash\{s\}$ и перейти к шагу 2.

Шаг 2. Объявить вершину $u$ помеченной. Выбрать в $\operatorname{th}(u, G)$ пороговый набор $\mathrm{Ch}(u)$ с номером count $(u)$, множество начальных вершин всех дуг из $\mathrm{Ch}(u)$ обозначить через $B(u)$. Положить $D P:=D P \cup \operatorname{Ch}(u), V P:=V P \cup B(u) \cup\{u\}$, $\operatorname{count}(u):=\operatorname{count}(u)+1$, и перейти к шагу 3 .

Шаг 3. Если все вершины в $V P$ помечены, то вывести минимальный путь $P$ с множеством вершин $V P$ и множеством дуг $D P$ и перейти к шагу 4. Если не все вершины в $V P$ помечены, то выбрать среди непомеченных вершин в $V P$ вершину $v$ наибольшего ранга. Положить $\operatorname{back}(v):=u, u:=v$, и перейти к шаry 2 . 
Шar 4. Выбрать в $\operatorname{th}(u, G)$ пороговый набор $\operatorname{Ch}(u)$ с номером $\operatorname{count}(u)-1$, множество начальных вершин дуг из $\mathrm{Ch}(u)$ обозначить через $B(u)$. Положить $D P:=D P \backslash \mathrm{Ch}(u), V P:=V P \backslash(B(u) \cup\{u\})$. Снять метку с вершины $u$. Перейти к шагу 5 .

Шаг 5. Если count $(u)>|\operatorname{th}(u, G)|$, то перейти к шагу 6. Если $\operatorname{count}(u) \leqslant|\operatorname{th}(u, G)|$, то перейти к шагу 2.

Шаг 6. Если $\operatorname{back}(u)=\varnothing$, то алгоритм завершает работу. Если $\operatorname{back}(u) \neq \varnothing$, то положить $u:=\operatorname{back}(u)$ и перейти к шагу 4 .

В силу леммы 1 данный алгоритм порождает множество $\mathscr{P}$ всех минимальных путей графа $G$, так как шаги 1-3 соответствуют процедуре $\operatorname{Proc}(s, \mathscr{L})$, а шаги 4-6 организуют перебор всех минимальных путей, основанный на принципе поиска в глубину [11]. Кроме того, временная сложность алгоритма равна $O(|D G| m)$, так как число операций между двумя последовательными выводами минимальных путей есть $O(|D G|)$.

Для дальнейшего понадобится определение сдвига некоторого множества $\mathscr{E}=$ $\left\{e_{i_{1}}, \ldots, e_{i_{k}}\right\}, i_{1}<\ldots<i_{k}$. Подмножество $\mathscr{E}^{\prime}=\left\{e_{j_{1}}, \ldots, e_{j_{k}}\right\}, j_{1}<\ldots<j_{k}$, называется левым сдвигом множества $\mathscr{E}$ (или $\mathscr{E}$ называется правым сдвигом $\mathscr{E}^{\prime}$ ), если $j_{1} \leqslant i_{1}, \ldots, j_{k} \leqslant i_{k}$. Предположим, что любой левый сдвиг сечения также является сечением. Тогда это равносильно тому, что любой левый сдвиг множества, содержащего некоторый минимальный путь из $\mathscr{P}$, также содержит некоторый минимальный путь из $\mathscr{P}$. Пары множеств $[D G, \mathscr{P}]$ с таким свойством известны как 2-монотонные системы [12], а соответствующая нумерация элементов из $D G$ называется регулярной [12].

Распознавание 2-монотонных систем и построение соответствующей регулярной нумерации осуществимы за время $O\left(\mathrm{~m}^{2}|D G|\right)[13]$. В [14] доказано, что 2-монотонные системы $[D G, \mathscr{P}]$ имеют $O(m|D G|)$ минимальных (относительно включения) сечений, которые могут быть определены за время $O(m|D G|)$.

Очевидно, что если для $G$ существует пороговая пара $(w, b)$, а дуги занумерованы в порядке невозрастания своих весов, то $[D G, \mathscr{P}]$ является 2-монотонной системой, т.е. 2-монотонность $[D G, \mathscr{P}]$ является необходимым условием для $G$ иметь пороговую живучесть. Считаем, что это условие выполняется для $G$.

Множество всех минимальных сечений в $G$ обозначим через $R=\left\{R_{1}, \ldots, R_{n}\right\}$. Пусть ind $(\mathscr{E})$ обозначает множество индексов дуг из $\mathscr{E}$. Положим $x_{i}=w\left(e_{i}\right), D G=$ $\left\{e_{1}, \ldots, e_{r}\right\}, x_{r+1}=b$. Тогда, по определению пороговой пары $(w, b)$, выполняются следующие неравенства:

$$
\begin{aligned}
\sum_{j \in i n d\left(R_{i}\right)} x_{j}-x_{r+1} \geqslant 0, & i=1, \ldots, n, \\
\sum_{j \in \text { ind }\left(D G \backslash P_{i}\right)} x_{j}-x_{r+1}<0, & i=1, \ldots, m \\
x_{i} & >0, \quad i=1, \ldots, r+1 .
\end{aligned}
$$

Обратно, любое решение системы (3) соответствует пороговой паре. Это вытекает из следующих двух фактов: любое сечение содержит некоторое минимальное сечение, произвольное множество $\mathscr{E}$, не являющееся сечением, содержится в некотором множестве $D G \backslash P_{i}$. 
Но совместность систем линейных неравенств является полиномиально разрешимой задачей [15], т.е. совместность системы (3) может быть определена за время, полиномиально зависящее от $m=|\mathscr{P}|$ и $r=|D G|$. Теорема доказана.

\section{Список литературы}

1. Черняк А.А., Комбинаторно-графовый метод анализа надежности сложных систем с положительными булевыми функциями. Автоматика и телемеханика (1991) №4, 165175 .

2. Chernyak A.A., A new graph-combinatorial method for reliability analysis of monotone graphs. In: Proc. 8th Intern. Symposium on Reliability in Radioelectronics. Budapest, 1991, pp. 135-140.

3. Chernyak A.A., Chernyak Zh.A., A unified domination approach for reliability analysis for networks with arbitrary logic in vertices. IEEE Trans. Reliab. (1996) 45, 114-119.

4. Satyanarayana A., Unified formula for analysis of some network reliability problems. IEEE Trans. Reliab. (1982) 31, 23-32.

5. Satyanarayana A., Hagstorm J.N., A new algorithm for the reliability analysis of multiterminal networks. IEEE Trans. Reliab. (1981) 30, 325-334.

6. Байхельт Ф., Франкен П., Надежность: математический подход. Радио и связь, Москва, 1988.

7. Черняк А.А., Ациклические монотонные графы: доминирование и надежность. Весці НАН Беларусі, Сер. б.--т.н. (1998) №3, 108-114.

8. Гэри М., Джонсон Д., Вычислителънъе машинъ и труднорешаемые задачи. Мир, Москва, 1982.

9. Иванов М.В., Можаев А.С., Рябинин Н.А., Вопросы судостроения. Судовая автомаmuxa (1984) 10, 18-24.

10. Hammer P.L., Maffrey F., Queyranne M., Cut-threshold graphs. Discrete Appl. Math. (1991) 30, 163-179.

11. Рейнгольд Э., Нивергельт Ю., Део Н., Комбинаторные алгоритмы: теория и практижа. Мир, Москва, 1980.

12. Sheng C.L., Threshold logic. Academic Press, New York, 1969.

13. Hammer P.L., Peled U.N., Pollaczek F., An algorithm to dualize a regular switching function. IEEE Trans. Comput. (1979) 28, 238-243.

14. Черняк А. А., Черняк ЖК. А., Надежность бинарных систем. Дискретная математиха (1999) 11, №1, 129-139.

15. Схрейвер А., Теория линейного и целочисленного программирования. Мир, Москва, 1991. 